\title{
Time-Resolved Contrast-Enhanced MR Angiography of Spinal Vascular Malformations
}

\author{
M. Amarouche, J.L. Hart, A. Siddiqui, T. Hampton, and D.C. Walsh
}

\begin{abstract}
BACKGROUND AND PURPOSE: The diagnosis of spinal vascular malformations may be challenging on conventional MR imaging because neither the location of the signal abnormality in the spinal cord nor the level of the abnormal flow voids correlates with the level of the fistula. We conducted a retrospective evaluation of the utility of using a time-resolved imaging of contrast kinetics sequence in the diagnosis, characterization, and localization of spinal vascular malformations, comparing it with the criterion standard of spinal DSA.
\end{abstract}

MATERIALS AND METHODS: Fifty-five consecutive patients with a suspected diagnosis of spinal vascular malformation underwent time-resolved imaging of contrast kinetics followed by spinal DSA. All scans were performed on a 1.5T scanner by using a standard 8-channel spine coil and were reported by a neuroradiologist before the DSA was performed.

RESULTS: Forty-seven lesions were confirmed on time-resolved imaging of contrast kinetics and classified as spinal dural arteriovenous fistulas ( $n=33$, with 1 patient having a type lb fistula), perimedullary spinal cord arteriovenous fistulas $(n=10)$, and intramedullary arteriovenous malformations $(n=3)$. One patient had an extradural spinal vascular malformation. Time-resolved imaging of contrast kinetics identified the location of the arterial feeder to within 1 vertebral level in $27 / 33$ patients (81.8\%) with spinal dural arteriovenous fistulas and correctly predicted the side in 22/33 (66.6\%) patients. Perimedullary spinal cord arteriovenous fistulas were erroneously considered to represent spinal dural arteriovenous fistulas before spinal DSA. The anatomy of the arterial supply to intramedullary arteriovenous malformations was also poorly characterized on time-resolved contrast-enhanced MR angiography.

CONCLUSIONS: It has been our experience that time-resolved imaging of contrast kinetics is a useful confirmatory tool when a spinal vascular malformation is suspected on the basis of clinical and conventional MR imaging findings. As experience with the technique grows and sequences are refined, it may be possible to rely on time-resolved imaging of contrast kinetics as a screening tool for the diagnosis of spinal vascular malformations.

ABBREVIATIONS: PMAVF = perimedullary spinal cord arteriovenous fistula; SCAVM = intramedullary arteriovenous malformation; SDAVF $=$ spinal dural arteriovenous fistula; SVM = spinal vascular malformation; TRICKS = time-resolved imaging of contrast kinetics; TR-MRA = time-resolved contrast-enhanced MR angiography

S pinal vascular malformations (SVMs) are an uncommon and heterogeneous group of vascular abnormalities. ${ }^{1-4}$ Early diagnosis is important because SVMs are a treatable cause of a potentially reversible myelopathy. Unfortunately, there is often considerable delay between symptom onset and treatment: The diagnosis of SVM may be challenging on conventional MR

Received April 3, 2014; accepted after revision July 2.

From the Departments of Neurosurgery (M.A., D.C.W.) and Neuroradiology (J.L.H., A.S., T.H.), King's College National Health Service Foundation Trust, London, United Kingdom; and Department of Clinical Neurosciences (D.C.W.), Institute of Psychiatry, King's College, London, United Kingdom.

Please address correspondence to Jonathan L. Hart, MD, King's College National Health Service Foundation Trust, Denmark Hill, London SE5 9RS, United Kingdom; e-mail: jonathanhart@nhs.net

三 Indicates article with supplemental on-line table.

http://dx.doi.org/10.3174/ajnr.A4164 imaging because the associated spinal cord intramedullary T2WI hyperintensity may mimic a tumor, an inflammatory pathology, or an infection. Moreover, neither the location of the signal abnormality in the spinal cord nor the level of the abnormal flow voids correlates with the level of the fistula.

Catheter-based spinal digital subtraction angiography is considered the criterion standard diagnostic technique to describe the angioarchitecture of SVMs. However, it is an invasive and potentially lengthy undertaking, requiring selective catheterization of all the arteries supplying the spinal cord. It will sometimes require general anesthesia to ensure that the patient is still during the procedure. If not, it may require staging to a second examination. The contrast and radiation doses can be higher than preferable.

It is therefore desirable that a noninvasive imaging technique to characterize these lesions either replace or allow targeted conventional angiography of SVMs. Time-resolved contrast- 
enhanced MR angiography (TR-MRA) techniques have shown particular promise. In this article, we describe their application in our institution to SVMs during a 7-year interval.

First described in the 1980s, ${ }^{5}$ TR-MRA techniques allowed the acquisition of images with good spatial and contrast resolution, but they were too slow to follow the uptake of the contrast agents, resulting in poorer temporal resolution images than DSA. The keyhole method proposed by van Vaals et $\mathrm{al}^{6}$ in 1993 accelerated the contrast uptake severalfold, resulting in better quality images. Three years later, Korosec et $\mathrm{al}^{7}$ introduced the time-resolved imaging of contrast kinetics (TRICKS). They described a method in which the $k$-space is sampled more frequently than the peripheral regions and timeframes are formed by temporal interpolation, continuously updating the spatial frequency uptake and significantly improving the image quality compared with the keyhole technique. TR-MRA techniques continue to progress with the most recent TRICKS techniques offering the ability to temporally resolve the arterial and venous phases of an MRA study. These are being used increasingly for evaluation of the vasculature in the extremities, ${ }^{8,9}$ brain, ${ }^{10,11}$ and spine. ${ }^{11,12}$

Our institution adopted TR-MRA by using the TRICKS sequence as part of our standard evaluation of suspected SVMs in 2005. We conducted a retrospective evaluation of the utility of using the TRICKS technique in the diagnosis, characterization, and localization of SVMs, comparing it with the criterion standard of spinal DSA.

\section{MATERIALS AND METHODS Patient Population}

Between May 2006 and January 2014, 55 patients with a suspected diagnosis of SVM (on clinical and conventional MR imaging grounds) underwent TRICKS followed by spinal conventional angiography.

Five patients were excluded from the study because both TRICKS and DSA had negative findings. The final clinical diagnosis in these patients was transverse myelitis, spinal cord tumor, spinal cord cavernoma, and idiopathic superficial siderosis. In 1 case, no definitive diagnosis was made (though an inflammatory cause was strongly suspected). DSA was unsuccessful in 4 patients due to technical difficulties, mainly related to aortoiliac ectasia and tortuosity, which precluded an adequate examination. These cases were explored surgically, guided by the TRICKS imaging and, in 1 case, additional CTA imaging.

Conventional MR imaging was performed without contrast in $57 \%$ of the remaining 50 patients. The diagnosis of SVM was suspected on initial MR imaging in $88 \%$ of patients.

\section{MR Imaging Technique}

All scans were performed on a Signa 1.5T scanner (GE Healthcare, Milwaukee, Wisconsin) by using a standard 8-channel spine coil. A counting whole-spine sagittal T1WI was performed followed by a sagittal T2WI on which a coronal 4 - to $5-\mathrm{cm}$ slab was positioned covering the vertebral body and spinal canal. The image volume was centered on the lower thoracic level to cover T6 to L2. This was modified in some circumstances when spinal cord swelling and signal change were located outside this standard FOV or focal hemorrhage was present (eg, in the context of intramedullary ar- teriovenous malformation [SCAVM] or perimedullary spinal cord arteriovenous fistula [PMAVF]). In cases in which the point of fistulation was outside the FOV, diagnostic confirmation was nevertheless obtained on the basis of identification of filling of median spinal cord veins in the arterial phase.

The TRICKS sequence parameters were FOV $=34$ (length/ craniocaudal), 3.2-mm section thickness, $1.6-\mathrm{mm}$ section gap, $256 \times 128$ matrix, $\mathrm{NEX}=1, \mathrm{TR}=3.14 \mathrm{~ms}, \mathrm{TE}=0.8 \mathrm{~ms}$. Twentyseven phases were obtained with 14 scan locations in each phase. The total scan time was 59 seconds with a temporal resolution of 1.8 seconds.

Following the acquisition of a mask, $20 \mathrm{~mL}$ of gadoliniumbased contrast agent was administered intravenously at $3 \mathrm{~mL}$ per second by a pump injector followed by a $50-\mathrm{mL}$ saline bolus "chaser." Triggering was performed manually after the mask acquisition with minimal or no delay.

\section{Catheter Angiography}

Selective intra-arterial spinal DSA was performed via a femoral approach in a dedicated biplanar neuroangiographic suite within 7 days of the MR imaging examination. Procedures were performed with the patient under local anesthetic. Iodinated nonionic contrast medium (3-5 mL of $300 \mathrm{mg} / \mathrm{mL}$ ) was injected selectively into the intercostal or lumbar radicular artery, expected to be supplying the lesion on the basis of TR-MRA findings, and into the contralateral radicular artery at the same level. Further bilateral injections were performed to include 2 levels above and below the identified fistula. If a fistula was not identified at the anticipated level, complete spinal angiography was undertaken, usually with the patient under general anesthesia.

Imaging was performed in the anteroposterior plane at 3 frames per second. Oblique, lateral, and magnified views were obtained as necessary to fully delineate the anatomy of the fistula.

\section{Image Processing and Review}

Fully automated postprocessed images were available for review as in-line subtracted coronal 3.2-mm datasets for each of the 27 MRA phases acquired and also as coronal maximum intensity projections. 3D volume reconstructions for the phases with maximal lesional conspicuity were also generated.

MR imaging and TRICKS scans were all reported by a neuroradiologist before DSA was performed. TRICKS findings were deemed positive if there was early filling of abnormal tortuous midline vessels (representing enlarged intradural longitudinal veins). The level of the feeding vessel was identified by the site of the earliest appearance of the abnormal midline vessel and by the lateral connection of the earliest appearing vessels to the radiculomedullary or segmental branches.

For this retrospective review, TRICKS findings were correlated with the subsequent angiographic results to assess the accuracy of the technique in identifying, characterizing, and localizing SVMs.

\section{Classification of SVM}

Several classification schemes have been described on the basis of the anatomic characteristics of these lesions or emphasizing their hemodynamic proprieties. ${ }^{1-4}$ One of the most widely used classi- 
fications ${ }^{4}$ groups these lesions into 4 different categories (summarized in Table 1): spinal dural arteriovenous fistulas (type I), intramedullary arteriovenous malformations (type II), extensive juvenile or metameric malformations (type III), and perimedullary spinal cord arteriovenous fistulas (type IV). SDAVFs are the most commonly identified type, comprising approximately $80 \%$ of SVMs. They are usually located on a nerve root sleeve within the neural foramen and are supplied by a dural branch of the segmental radicular artery.

\section{RESULTS}

Of 50 patients (age range, 5-83 years; median, 61 years; 24 females, 26 males), 46 had both TRICKS-MRA and DSA available for comparison and 4 were managed on the basis of TRICKS findings with additional CTA findings in 1 patient. The suspected diagnosis of SVM, based on the finding of early-filling, median longitudinal venous structures on TRICKS, was confirmed with DSA in 41 cases. In 3 cases, TRICKS failed to show an SVM that was subsequently identified by DSA. In 3 other cases, the initial TRICKS was reported as suggestive of an SVM but subsequent comprehensive spinal angiography failed to demonstrate an arteriovenous shunt. The final diagnosis for these patients was subsequently reported as long-standing arachnoiditis with syrinx and primary spinal cord neoplasm in 2 patients. The third patient had repeat TRICKS 4 months after the initial study showing no arteriovenous shunting. Including all 54 studies, sensitivity, specificity, positive predictive value, and negative predictive value for the

\begin{tabular}{|c|c|c|}
\hline Type & Description & No. \\
\hline I & $\begin{array}{l}\text { SDAVF: } \\
\text { la) Single fistula } \\
\text { lb) Polyfistulous }\end{array}$ & 33 \\
\hline II & SCAVM & 3 \\
\hline III & Juvenile/metameric malformations & 0 \\
\hline \multirow[t]{4}{*}{ IV } & PMAVF $^{13}$ & 10 \\
\hline & A) Single fistula & \\
\hline & B) Often multiple, intermediate flow & \\
\hline & C) Polyfistulous, large lesions & \\
\hline
\end{tabular}

detection of a spinal vascular malformation on TRICKS in comparison with the criterion standard DSA were 98\%, 63\%, 93\%, and $83 \%$, respectively. The low negative predictive value is probably due to a low data sample of studies with negative findings.

The 47 lesions confirmed on TRICKS were classified as SDAVF ( $n=33$, with 1 patient having a type Ib fistula), PMAVF $(n=10)$, and SCAVM $(n=3)$ (Table 1$)$. One patient had an extradural SVM. Patient imaging findings are recorded in the Online Table and Table 2.

In the SDAVF group $(33 / 47,70.2 \%)$, the segmental level of fistulation identified on TRICKS was concordant with conventional angiography findings in 21 of 33 patients (63.6\%) (Fig 1). In 6 patients, it was within 1 predicted level, and in 3 patients, within 2 predicted levels. The correct side was predicted in 22 of 33 patients (66.6\%). One patient with an SDAVF had cervical spinal cord signal change on MR imaging and was found to have a posterior fossa fistula with reflux into cervical spinal cord veins. In this case, cervical spine intramedullary signal change was evident. The TRICKS was centered on this level and duly confirmed filling of median spinal veins in the arterial phase. The arterial supply to the fistula was not evident on this acquisition and was delineated on DSA.

In the PMAVF group $(10 / 47,21.27 \%)$, TRICKS and the DSA level were concordant in 1 patient, within 1 predicted level in 2 patients, and within 2 predicted levels in 3 patients (Fig 2). The correct side was identified in 2 patients. Additional feeding vessels were also demonstrated on DSA in 2 of 3 patients with PMAVFs; these lesions were also initially erroneously characterized as SDAVFs following TRICKS.

In the SCAVM group $(3 / 47,6.38 \%)$, the nidus was accurately located on TRICKS and the extent of draining veins was demonstrated in 1 patient (Fig 3). However, feeding vessels were not identified and characterized reliably: Conventional angiography confirmed the presence of multiple feeders in these lesions, 2 of which had not been resolved on TRICKS. In the case of the sacral fistula, TRICKS centered on T6 to L2 showed arterial phase filling of the spinal cord venous plexus. A feeder was suspected at L2 on TRICKS but was not seen on conventional angiography. Selective catheterization of the median sacral artery demonstrated the fistula.

Table 2: TRICKS, DSA findings, and treatment of patients with intramedullary arteriovenous malformations, perimedullary spinal cord arteriovenous fistulas, and extradural SVMs

\begin{tabular}{|c|c|c|c|c|c|}
\hline \multirow[b]{2}{*}{ Age (yr) } & \multirow[b]{2}{*}{ Sex } & \multicolumn{2}{|c|}{ Side and Level of Feeding Vessel } & \multirow[b]{2}{*}{ Treatment } & \multirow[b]{2}{*}{ Classification } \\
\hline & & TRICKS & Angiogram & & \\
\hline 24 & M & Conus midline & $\mathrm{LTT} 0$ & Surgery & II \\
\hline 46 & M & L C5/6 & $\mathrm{R} C 5 / 6$ & Awaiting radiosurgery & ॥ \\
\hline 58 & $\mathrm{~F}$ & $\begin{array}{l}\text { SVM but no level or side } \\
\text { identified }\end{array}$ & $\begin{array}{l}\text { Left vertebral artery just } \\
\text { beyond the PICA origin }\end{array}$ & Surgery & II \\
\hline 78 & M & $\mathrm{TII} / \mathrm{T} 12$, no side identified & L L1 & Surgery & IV \\
\hline 73 & M & R L3/L4 & R L1 & Surgery & IV \\
\hline 33 & M & R T11 & R T11 & Surgery & IV \\
\hline 71 & $\mathrm{~F}$ & $\begin{array}{l}\text { L L2, postembolization } \\
\text { to coccyx }\end{array}$ & $\begin{array}{l}\text { Distal coccyx, branches of the } \\
\text { median sacral artery }\end{array}$ & Multiple embolizations and surgery & IV \\
\hline 45 & M & R T6 & L T7 & Radiosurgery & IV \\
\hline 17 & M & T9, no side identified & L T7 & Awaiting radiosurgery & IV \\
\hline 82 & $\mathrm{~F}$ & Bilateral T12 & Unsuccessful & Surgery & IV \\
\hline 79 & $\mathrm{~F}$ & L T11 & Unsuccessful & Surgery & IV \\
\hline 74 & $\mathrm{~F}$ & R L3/L4 & Unsuccessful & Surgery & IV \\
\hline 32 & $\mathrm{~F}$ & No SVM identified & $\mathrm{R} C 2$ & Conservative & IV \\
\hline 65 & M & R L3/L4 & R L3/L4 & Embolization & Extradural \\
\hline
\end{tabular}

Note:- L indicates left; R, right; PICA, posterior inferior cerebellar artery. 

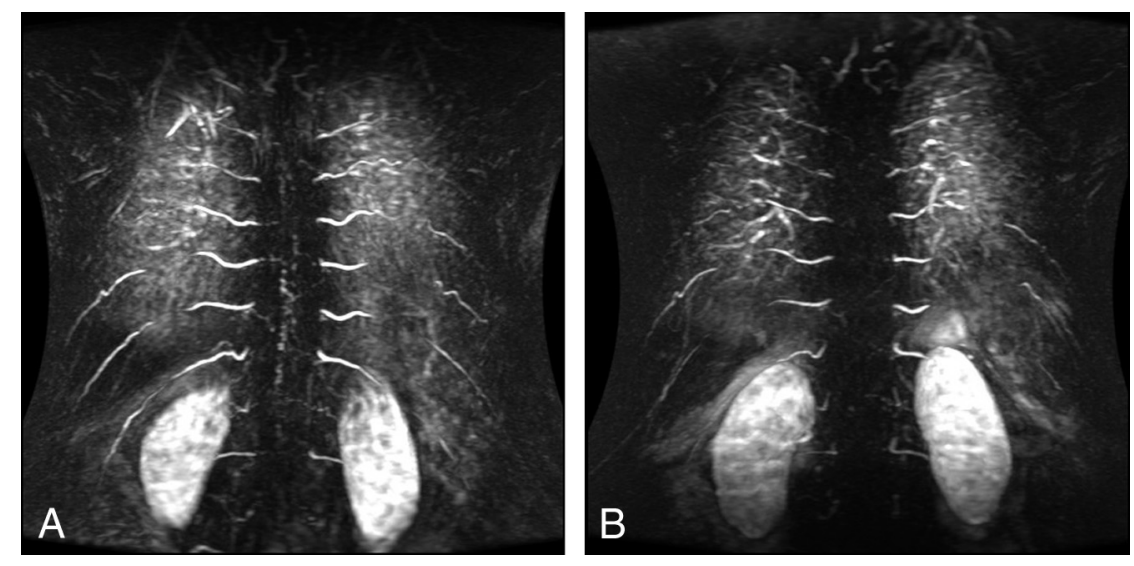

FIG 1. Preoperative $(A)$ and postoperative $(B)$ TRICKS sequences of a patient with an SDAVF (type I).
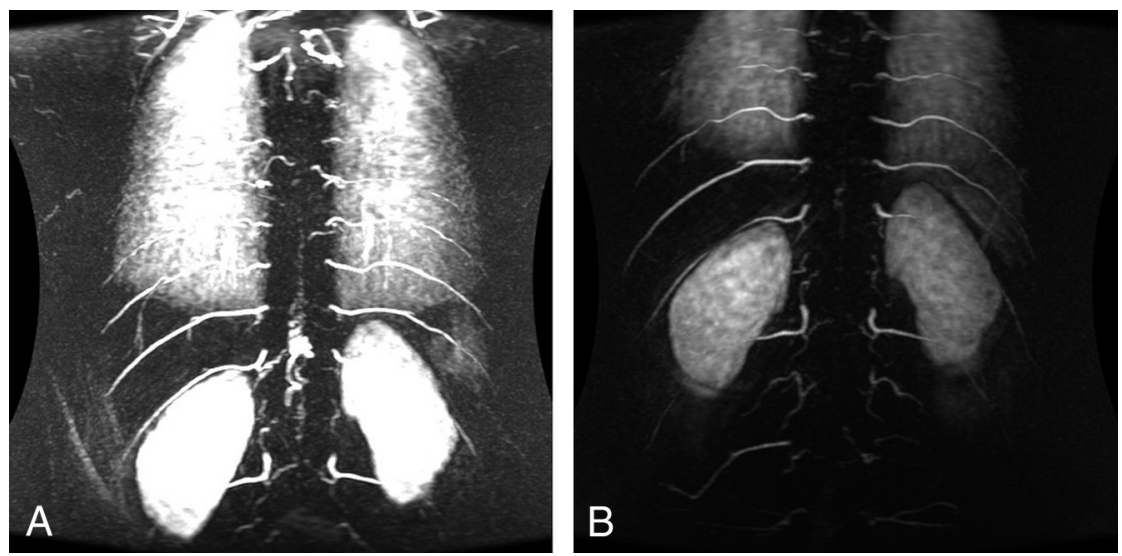

FIG 2. Preoperative (A) and postoperative (B) TRICKS sequences of a patient with a PMAVF (type IV).
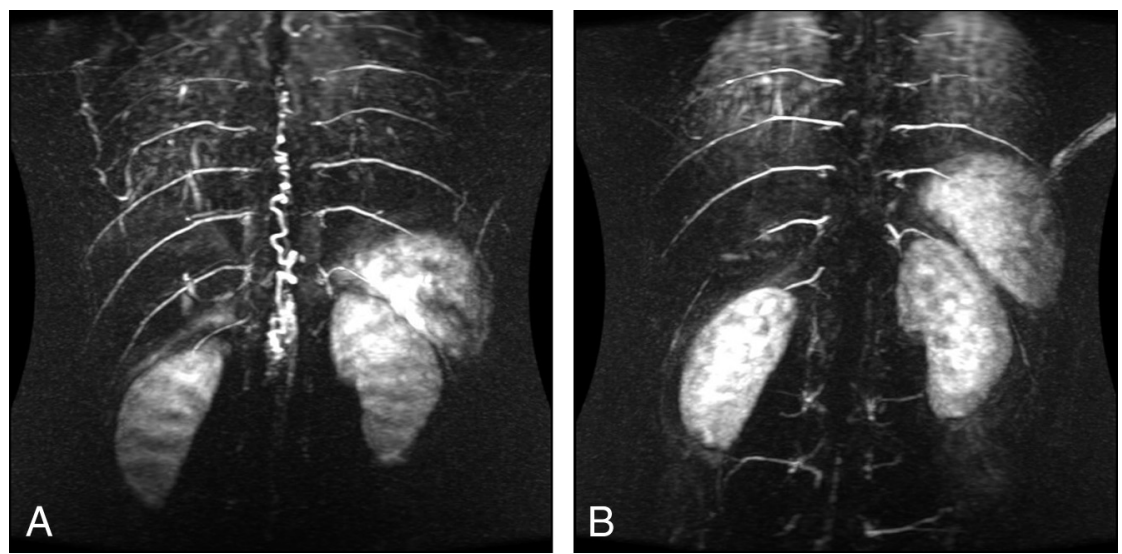

FIG 3. Preoperative $(A)$ and postoperative $(B)$ TRICKS sequences of a patient with SCAVM of the conus (type II).

Patients were treated surgically in most cases. Three patients underwent endovascular treatment, but 2 attempts at embolization withOnyx (Covidien, Irvine, California) of 1 sacral dural arteriovenous fistula were unsuccessful in closing all supplying arteries and surgical treatment was necessary to achieve complete obliteration of the fistula. In 3 cases of SCAVM, the lesion anatomy (in particular the relationship to a major anterior spinal artery radiculomedullary supply) precluded safe endovascular or surgical treatment. One patient has been treated by using radiosurgery, and 2 patients are awaiting the same treatment. One patient refused treatment and is being managed conservatively.

\section{DISCUSSION}

TRICKS is increasingly used for noninvasive vascular imaging. Fast, time-resolved imaging of the spine increases temporal resolution, permitting hemodynamic assessment of SVMs over a relatively large FOV (with relatively little compromise in spatial resolution or signal-to-noise ratio). The TRICKS sequence acquires central $k$-space data in all sections, but the remaining peripheral data are split and shared among sections. Combining this $k$-space data acquisition with a very low $\mathrm{TR}$, on the order of approximately $3 \mathrm{~ms}$, allows rapid data acquisition and provides dynamic, multiphase real-time angiographic information. The increased temporal resolution achieved is of particular value in the evaluation of arteriovenous shunts, and preliminary results have also been described in studies of intracranial AVMs and dural fistulas. ${ }^{10,11,14}$ Other advantages include a shorter acquisition time and lack of requirement for contrast bolus timing. ${ }^{15}$

Standard TOF MRA techniques lack sufficient temporal resolution to identify SVMs, and phase-contrast methods are not suited to the detection of these often relatively low-flow shunts. Contrast-enhanced MRA techniques with 3D TOF acquisition have been described by a number of authors, who report a reasonable degree of success in identifying the level of the SDAVF (on the order of 50\%-75\%). ${ }^{16-18}$ Elliptic-centric contrast-enhanced MRA 3D techniques have also proved useful in demonstrating the presence and level of SDAVFs, ${ }^{19,20}$ but again they do not demonstrate the actual fistula or resolve arterial and venous phases to allow differentiation of the fistula and draining radiculomedullary vein from the engorged coronal venous plexus. The level of fistula was inferred in these studies by tracing an engorged medullary vein retrogradely to the neural foramen. Without the ability to distinguish this vein from veins draining away from the coronal venous plexus, there is the potential for false localization. CTA is similarly limited by the inability to temporally resolve arteries and veins. ${ }^{21,22}$ Images with high spatial resolution can be obtained, but interpretation is hampered by the adjacent bony 
anatomy. In the recently published article by Saindane et al, ${ }^{15} \mathrm{a}$ higher number of studies with negative findings were reported, explaining the higher negative predictive value compared with our study.

Our results demonstrate that the TRICKS technique is reliable in confirming the presence of a suspected SVM, though there was a high pretest probability based on the clinical and MR imaging findings. As experience with the technique grows and sequences are refined, it may become possible to rely on TRICKS as a screening tool, but it cannot currently be depended on as a "rule-out" test in cases of a suspected SVM.

While the temporal resolution of the 2 techniques is not substantially different, the difference in spatial resolution remains considerable and largely accounts for the difficulty in distinguishing SDAVF from PMAVF and in adequately characterizing SCAVM with TRICKS. For SDAVF, TRICKS identified the location of the arterial feeder to within 1 vertebral level in 27 of 33 patients, and in most cases, a targeted spinal DSA (performed with the patient under local anesthetic) could be performed following TRICKS, minimizing radiation exposure and contrast dose. Cases of sacral SDAVF $(n=1)$ and rather small bilateral thoracic SDAVF $(n=1)$ necessitated more comprehensive DSA examination. The 3 cases of PMAVF were erroneously considered to represent SDAVF before spinal DSA, which also identified additional supplying arteries. The anatomy of the arterial supply to SCAVM was also poorly characterized on TRICKS, and we were unable to resolve the angioarchitecture of this type of SVM. On this basis, spinal DSA remains mandatory to accurately characterize SVMs. Conventional angiography also demonstrates the level of origin of the artery of Adamkiewicz or other radiculomedullary branches; this demonstration is essential when considering treatment of these lesions.

Interpretation of the technique requires some experience on the part of the reporting neuroradiologist, but common pitfalls can generally be avoided by the appropriate use of the cross-referencing tools and scrutinizing individual arterial phase datasets in addition to MIP images. Although 3 studies were initially reported as having positive findings, on retrospective review, no false-positive studies were identified. One case, reported early in the series, was due to misinterpretation of an extraspinal longitudinal venous structure as a median longitudinal spinal vein. The 2 other cases were rather equivocal, with no clear level of supply or fistulation to the potentially abnormal median veins identifiable on TRICKS. There was only 1 case in which TRICKS failed to identify a SVM. The patient had a high cervical PMAVF.

Our results are consistent with findings reported by a number of other investigators. Ali et $\mathrm{al}^{23}$ described the use of a TR-MRA (TRICKS) sequence to predict the level of SDAVF to within 1 vertebral level in 6 patients. The authors also suggested that the technique was accurate for excluding the presence of SDAVFs and in confirming successful fistula occlusion following treatment. Tapping et $\mathrm{al}^{12}$ described the use of the TRICKS sequence after a technically difficult spinal catheter angiogram (due to aortic tortuosity and atherosclerosis), which did not locate the anticipated SDAVF. It was subsequently located on 3T TR-MRA. In a second case, it was impossible to obtain a stable catheter position at the level of the known fistula; therefore, 3T TRICKS was used to fur- ther characterize the lesion before surgery. We have also encountered a small number of patients $(n=4)$ in whom adequate DSA could not be completed due to technical difficulties and who underwent surgery solely on the basis of information from the TRICKS.

Limitations of the study include the small number of patients having a rare condition and its retrospective design. Although images were analyzed by only a single neuroradiologist, assessment preceded the DSA in all cases; thus, the neuroradiologist was blinded to the nature and level of the lesion. Further evaluation of the technique might incorporate an assessment of interobserver agreement.

\section{CONCLUSIONS}

The infrequency of these cases precludes large studies. Consequently, conclusions have to be drawn from pooled case series similar to the present study. It has been our experience that TRICKS is a useful confirmatory tool when an SVM is suspected on the basis of clinical and conventional MR imaging findings. The frequent ability to identify the level of arteriovenous shunting on TRICKS allowed the subsequent spinal catheter angiography to be directed to the level of interest and involved less contrast load and radiation exposure; these features abbreviated an otherwise lengthy and potentially difficult procedure.

DSA remains necessary to characterize SVMs and, particularly in the case of PMAVF and SCAVM, to identify important radiculomedullary feeders before consideration of treatment.

\section{REFERENCES}

1. Black P. Spinal vascular malformations: an historical perspective. Neurosurg Focus 2006;21:E11

2. Spetzler RF, Detwiler PW, Riina HA, et al. Modified classification of spinal cord vascular lesions. J Neurosurg 2002;96:145-56

3. Qureshi AI. A new classification scheme for spinal vascular abnormalities based on angiographic features. J Neuroimaging 2013;23:401-08

4. Anson JA, Spetzler RF. Classification of spinal arteriovenous malformations and implications for treatment. BNI Quarterly 1992;8:2-8

5. Grist TM, Mistretta CA, Strother CM, et al. Time-resolved angiography: past, present, and future. J Magn Reson Imaging 2012;36:1273-86

6. van Vaals JJ, Brummer ME, Dixon WT, et al. "Keyhole" method for accelerating imaging of contrast agent uptake. J Magn Reson Imaging 1993;3:671-75

7. Korosec FR, Frayne R, Grist TM, et al. Time-resolved contrast-enhanced 3D MR angiography. Magn Reson Med 1996;36:345-51

8. Andreisek G, Pfammatter T, Goepfert K, et al. Peripheral arteries in diabetic patients: standard bolus-chase and time-resolved MR angiography. Radiology 2007;242:610-20

9. Ruhl KM, Katoh M, Langer S, et al. Time-resolved 3D MR angiography of the foot at $3 \mathrm{~T}$ in patients with peripheral arterial disease. $A J R$ Am J Roentgenol 2008; 190:W360-64

10. Petkova M, Gauvrit JY, Trystram D, et al. Three-dimensional dynamic time-resolved contrast-enhanced MRA using parallel imaging and a variable rate $k$-space sampling strategy in intracranial arteriovenous malformations. J Magn Reson Imaging 2009;29:7-12

11. Saleh RS, Lohan DG, Villablanca JP, et al. Assessment of craniospinal arteriovenous malformations at $3 \mathrm{~T}$ with highly temporally and highly spatially resolved contrast-enhanced MR angiography. AJNR Am J Neuroradiol 2008;29:1024-31

AJNR Am J Neuroradiol 36:417-22 Feb 2015 www.ajnr.org 421 
12. Tapping CR, Thackeray AT, Bartlett RJ, et al. Evaluation of spinal dural arteriovenous fistulae with gadofosveset trisodium at $3 \mathrm{~T}$ (TRICKS and LAVA). Clin Radiol 2011;66:865-68

13. Merland JJ, Reizine D. Embolization techniques in the spinal cord. In: Dondelinger RF, Rossi P, Kurdziel JC, et al, eds. Interventional Radiology. New York: Thieme; 1990:433-42

14. Kunishima K, Mori H, Itoh D, et al. Assessment of arteriovenous malformations with 3-Tesla time-resolved, contrast-enhanced, three-dimensional magnetic resonance angiography. J Neurosurg 2009;110:492-99

15. Saindane AM, Boddu SR, Tong FC, et al. Contrast-enhanced timeresolved MRA for pre-angiographic evaluation of suspected spinal dural arterial venous fistulas. J Neurointerv Surg 2015;7:135-40

16. Binkert CA, Kollias SS, Valavanis A. Spinal cord vascular disease: characterization with fast three-dimensional contrast-enhanced MR angiography. AJNR Am J Neuroradiol 1999;20:1785-93

17. Bowen BC, Fraser K, Kochan JP, et al. Spinal dural arteriovenous fistulas: evaluation with MR angiography. AJNR Am J Neuroradiol 1995; $16: 2029-43$

18. Saraf-Lavi E, Bowen BC, Quencer RM, et al. Detection of spinal dural arteriovenous fistulae with MR imaging and contrast-enhanced
MR angiography: sensitivity, specificity, and prediction of vertebral level. AJNR Am J Neuroradiol 2002;23:858-67

19. Farb RI, Kim JK, Willinsky RA, et al. Spinal dural arteriovenous fistula localization with a technique of first-pass gadoliniumenhanced MR angiography: initial experience. Radiology 2002;222:843-50

20. Luetmer PH, Lane JI, Gilbertson JR, et al. Preangiographic evaluation of spinal dural arteriovenous fistulas with elliptic centric contrast-enhanced MR angiography and effect on radiation dose and volume of iodinated contrast material. AJNR Am J Neuroradiol 2005;26:711-18

21. Lai PH, Weng MJ, Lee KW, et al. Multidetector CT angiography in diagnosing type I and type IVA spinal vascular malformations. AJNR Am J Neuroradiol 2006;27:813-17

22. Terae S, Kudo K, Asano T, et al. CT angiography with multidetectorrow helical CT in spinal arteriovenous malformation. Clin Imaging 2004;28:23-27

23. Ali S, Cashen TA, Carroll TJ, et al. Time-resolved spinal MR angiography: initial clinical experience in the evaluation of spinal arteriovenous shunts. AJNR Am J Neuroradiol 2007;28:1806-10 